\title{
Dossier: Trabajo y sociedad en América Latina: Introducción
}

\author{
Julio César Neffa ${ }^{1}$ y Pablo Barbetti ${ }^{2}$
}

La problemática del trabajo humano ha sufrido grandes transformaciones a nivel global desde mediados de la década de 1970. Y esa dinámica continúa en nuestros días.

Por una parte, desde la emergencia de la crisis en esa época y junto con el estancamiento económico, termina en los países capitalistas industrializados el período del pleno empleo y el incremento real de los salarios directos e indirectos. Esta situación se expandió en el mundo por el impulso de las empresas transnacionales y por la implementación de las políticas neoliberales. Estas últimas provocaron un fuerte ajuste de las economías latinoamericanas ya que gran parte de sus países orientó sus políticas a partir de consignas del Consenso de Washington: privatización de las empresas públicas y del sistema de seguridad social -AFJP y ART en Argentina-, apertura indiscriminada al comercio exterior, revalorización del papel del mercado para la asignación de recursos, desregulación de los mercados -con excepción del mercado de trabajo, donde las reformas laborales se orientaron a la flexibilización del uso de la fuerza de trabajo y la reducción del costo salarial, y de las contribuciones al sistema de seguridad social-. Estas medidas, que se impusieron en paralelo con el desempleo provocado por el cierre de empresas, contribuyeron al incremento de la pobreza y de la indigencia, dando lugar a una creciente desigualdad y al debilitamiento de los sindicatos.

\footnotetext{
${ }^{1}$ CEIL-PIETTE- CONICET

${ }^{2}$ CES- UNNE
} 
De Prácticas y discursos/ Universidad Nacional del Nordeste/ Centro de Estudios Sociales

Al mismo tiempo, termina un ciclo denominado "sociedad salarial"; además del incremento del desempleo, aumenta la proporción de los trabajadores no registrados y los trabajadores precarios -por tiempo determinado, contratados por medio de empresas de servicios eventuales o bajo otras figuras como las pasantías, los empleos de planta transitoria, los monotributistas y los autónomos, etc-.

Sin embargo, a pesar de estas profundas transformaciones, que en la región se presentan con una gran heterogeneidad y fuertes diferencias con relación a la situación en los países capitalistas industrializados, se observa que el trabajo no ha perdido centralidad.

Este sexto número de la revista De prácticas y discursos. Cuadernos en Ciencias Sociales está dedicado a visualizar producciones del campo académico, provenientes de las ciencias sociales, que abordan el análisis de estos cambios en contextos específicos en América Latina. La respuesta a la convocatoria realizada, que consideramos altamente satisfactoria, deriva en esta entrega que reúne once artículos de autores provenientes de diversas universidades y centros de investigación de Argentina, que entendemos contribuyen a problematizar distintos objetos de conocimiento sobre el fenómeno del trabajo humano y sus modificaciones recientes, en distintos territorios.

Javier Alegre, en su artículo Desigualdad y Trabajo: un camino a revertir, analiza el crecimiento de las desigualdades socio-económicas a escala global, que se dio en las últimas décadas, mostrando rasgos y consecuencias de este proceso en la esfera laboral, a la vez que realiza un recorrido por los principales aportes conceptuales dirigidos a precisar el modo en que el trabajo puede ser entendido como fuente de mayores niveles de igualdad.

Por su parte, Pablo Bonfiglio y Guillermina Comas analizan el objeto que enuncian en el título de su artículo: Procesos de reproducción social e integración sociolaboral. Trayectorias de inserción al mercado laboral de trabajadores del Conurbano bonaerense. Para ello, recurren al análisis de las trayectorias de inserción al mercado de trabajo y de los significados atribuidos a la tarea de trabajadores residentes en un espacio territorialmente segregado y con altos niveles de informalidad laboral, situado en la periferia del Conurbano bonaerense. Los datos que aportan los autores muestran 
De Prácticas y discursos/ Universidad Nacional del Nordeste/ Centro de Estudios Sociales

una disminución en las chances de movilidad en las trayectorias laborales de ingreso al mercado y un endurecimiento del origen social sobre las posibilidades de calidad del recorrido laboral. Sin embargo, señalan que esto no se tradujo necesariamente en el plano de las representaciones y expectativas subjetivas con relación a su situación laboral y a las chances de movilidad.

Las respuestas encaradas desde los Estados, así como los debates actuales sobre las políticas sociales que surgieron para hacer frente a algunas de estas trasformaciones, combatir la pobreza y dar respuesta a los recientes procesos de desigualdad social en nuestra región, son abordados, específicamente, en dos artículos.

Lucas Torrice, en su trabajo ¿Estamos todos de acuerdo con la Asignación Universal por Hijo? Entre el liberalismo y la recuperación del Estado, expone una serie de herramientas conceptuales que posibilita comprender el actual consenso de todo el espectro político en torno a una política social como la Asignación Universal por Hijo $(\mathrm{AUH})$. Con esa finalidad, reconstruye los contextos y antecedentes de la AUH y las diversas justificaciones que se pueden encontrar en distintas tradiciones teóricopolíticas. En tal sentido, el autor plantea que "desde distintos posicionamientos ideológicos se puede brindar un consentimiento a la $\mathrm{AUH}$, así sea vista únicamente como una política de asistencia focalizada en los grupos más vulnerables para paliar la pobreza, como que se la valore como un piso de ingresos que apunta a garantizar un ingreso "universal" para la niñez".

El proceso de reconfiguración de la protección social -workfare-, entendido como una nueva forma de gestionar los riesgos sociales mediante la implementación de políticas sociales y de empleo está también presente en el trabajo de Brenda Brown titulado ¿La condicionalidad como nuevo paradigma de política social en América Latina?. La autora avanza en una caracterización de este sistema de protección social en América Latina. Para ello realiza una comparación de distintos Programas de Transferencias Monetarias Condicionadas implementados en Latinoamérica y analiza el diagnóstico en el que estos se sustentan, las causas del consenso generalizado de su implementación y distingue tres tipos de PTMC que se diferencian por el grupo objetivo al que se dirigen y por el problema social sobre el que se focalizan -pobreza, desempleo o informalidad-. 
Los espacios de trabajos auto gestionados constituyen, también, otros ámbitos donde ponen la mirada algunas producciones de este dossier. Específicamente dos artículos problematizan diferentes aspectos en los mismos.

Uno de ellos es el artículo Discursos mediáticos y trabajo auto gestionado: ¿Cómo se representó el proceso de recuperación de fábricas luego de la crisis del 2001 y diez años después? escrito por Vanesa Coscia. En esta producción, se abordan los procesos de ocupación/recuperación de fábricas autogestionadas en Argentina, desde el análisis interdisciplinario de los Estudios Culturales, articulando estudios sobre los medios masivos de comunicación y del trabajo. Específicamente, la autora analiza de qué modo se otorgó visibilidad, desde los principales diarios de circulación masiva de Argentina -Clarín y La Nación-, a este tipo de procesos en dos períodos históricos: 2002/2004 y 2010/2013. La escritora plantea que el análisis de las representaciones mediáticas sobre el mundo laboral invita a reflexionar sobre el modo en que se somete a consideración pública la problemática del trabajo en sociedades mediatizadas.

Amalia Miano, David Burín y Ana Inés Heras Monner Sans, en cambio, centran su análisis en el rol asignado a la tecnología en cooperativas de trabajo autogestionadas ubicadas en la provincia de Buenos Aires y Santa Fe. Una de las conclusiones de su trabajo, Tecnología y autogestión en cooperativas de trabajo, es que las cooperativas de trabajo, como forma de organización social y productiva diferente a aquellas que tienen al capital como eje organizador, a través de determinados mecanismos específicos -igualdad entre los miembros, posibilidad de tomar decisiones por parte de todos los trabajadores, orientaciones hacia la justicia, dispersión del poder-, posibilitan otras formas de interactuar e implementar tecnologías.

El artículo de María Laura Henry, por su parte, constituye una contribución en un campo de análisis diferente que despierta un creciente y renovado interés: el trabajo independiente cuentapropista. En su artículo, denominado Perfiles y especificidades del trabajo independiente cuentapropista en el escenario productivo actual. Un contrapunto entre los periodistas colaboradores de prensa y los diseñadores de 
De Prácticas y discursos/ Universidad Nacional del Nordeste/ Centro de Estudios Sociales

indumentaria independientes, realiza una caracterización de las condiciones laborales de dos grupos de trabajadores: los periodistas colaboradores y los diseñadores de indumentaria independientes, identificando factores explicativos y las ventajas y/o desventajas que conlleva para los trabajadores en cada caso esta modalidad.

Los siguientes dos artículos de esta presentación tienen como punto de contacto el hecho de que abordan algunas dimensiones vinculads al trabajo en el ámbito estatal.

El primero de ellos se denomina Acerca de la dimensión histórico-política del trabajo en clave estatal. Gestión de la Fuerza de Trabajo en la ¿burocracia? provincial correntina y fue escrito por Valeria Ojeda. En esta producción, la autora distingue algunos aspectos relevantes que conforman la dimensión histórico-política del trabajo en el Estado provincial correntino. A partir de un análisis que pone en relieve las características del sistema de gestión de la fuerza de trabajo estatal, discute cómo tales elementos influyen, impactan y van otorgando diversidad de sentido a la gestión de los trabajadores.

Por su parte, la comprensión del proceso de trabajo en instituciones estatales que albergan a jóvenes en conflicto con la ley es el objetivo general que se proponen Patricia Gorri y Patricia Lecano, en su artículo Trabajo y conflicto social. Los operadores en el Sistema de Responsabilidad Penal Juvenil de Mendoza, Argentina. Las autoras se preguntan qué implica ser trabajador en una institución cerrada, cuáles son las dinámicas y prácticas laborales, y de qué manera el conflicto social y el rol represor de estas instituciones se hace visible en el discurso y las prácticas de los trabajadores que cotidianamente conviven con jóvenes transgresores. Para ello, parten del análisis de un conflicto específico producido a fines de 2012, en esa provincia, que afectó de manera directa a los operadores del sistema.

Carlos Andelique y María Cecilia Tonón, en Una aproximación a las acciones colectivas de los docentes santafesinos frente a las políticas educativas (1983-1989). El caso de la Asociación de Magisterio de Santa Fe (AMSAFE), también abordan la conflictividad de un colectivo de trabajadores del Estado, pero desde otro enfoque. A lo largo del texto presentan un análisis de las características que asumieron las acciones colectivas llevadas a cabo por los docentes santafesinos nucleados en AMSAFE, entre 1983 y 
De Prácticas y discursos/ Universidad Nacional del Nordeste/ Centro de Estudios Sociales

1989, y plantean algunas conjeturas sobre las posibles causas que explican estos procesos de conflictividad que se manifestaron en el período histórico particular, coincidente con el primer gobierno posdictadura.

Un último artículo que forma parte del dossier es De sirvientas a trabajadoras domésticas. Nuevas configuraciones del servicio doméstico en Corrientes, de Santiago Canevaro. Este trabajo se propone analizar las nuevas configuraciones del servicio doméstico en la ciudad de Corrientes, considerando los procesos de transformación que vienen ocurriendo en esta ocupación en las últimas décadas -entre ellos, el pasaje de la modalidad "sin retiro" a "con retiro" y sus efectos sobre las relaciones que se establecen entre empleadas domésticas y los empleadores, los cambios en los patrones migratorios de las mujeres en el servicio doméstico, la ampliación de derechos sociales hacia las poblaciones de sectores populares, así como el aumento del nivel de instrucción de las empleadas domésticas más jóvenes-. Así, recurriendo a la utilización de herramientas cualitativas, el autor analiza las experiencias y trayectorias laborales de las empleadas domésticas de esta ciudad, indagando en sus concepciones y sentidos ligados al trabajo doméstico y a las perspectivas de futuro, exhibiendo la heterogeneidad de situaciones que se establecen en las relaciones cotidianas con sus empleadores.

Los distintos artículos aquí reunidos permiten advertir con claridad las profundas transformaciones a las que hacíamos referencia al inicio de la presentación, en el plano macro-estructural, así como sus derivaciones tanto en el plano de las políticas públicas como en su incidencia a nivel micro-organizacional en los procesos de trabajo y sus modos de gestión.

Por otra parte, la diversidad de temas y enfoques de estas producciones, a la vez que muestran la heterogeneidad de situaciones en la estructura social argentina, ponen de relieve la vigencia del valor del trabajo humano como una actividad que sigue constituyendo un esfuerzo creativo que -además de posibilitar la sobrevivencia en forma autónoma- expresa la subjetividad del trabajador, juega un papel importante en el desarrollo de la personalidad, construye un colectivo de trabajo y contribuye a construir una identidad profesional. 
De Prácticas y discursos/ Universidad Nacional del Nordeste/ Centro de Estudios Sociales

Año 5, Número 6, 2016 (Enero - Julio)

ISSN 2250-6942

En suma, los diferentes trabajos aportan hallazgos de valor y abren nuevos interrogantes. Esperamos, en tal sentido, que este número bibliográfico contribuya a estimular y profundizar los debates, análisis y producciones en este campo. 